\title{
General criterion for oblivious remote state preparation
}

\author{
Z. Kurucz, ${ }^{1}$ P. Adam, ${ }^{1,2}$ and J. Janszky ${ }^{1,2}$ \\ ${ }^{1}$ Department of Nonlinear and Quantum Optics, Research Institute for Solid State Physics and Optics, \\ Hungarian Academy of Sciences, P.O. Box 49 H-1525 Budapest, Hungary \\ ${ }^{2}$ Research Group for Nonlinear and Quantum Optics, \\ Hungarian Academy of Sciences and Institute of Physics, \\ University of Pécs, Ifjúság út 6. H-7624 Pécs, Hungary
}

(Dated: 8 June, 2005)

\begin{abstract}
A necessary and sufficient condition is given for general exact remote state preparation (RSP) protocols to be oblivious, that is, no information about the target state can be retrieved from the classical message. A novel criterion in terms of commutation relations is also derived for the existence of deterministic exact protocols in which Alice's measurement eigenstates are related to each other by fixed linear operators similar to Bob's unitaries. For non-maximally entangled resources, it provides an easy way to search for RSP protocols. As an example, we show how to reduce the case of partially entangled resources to that of maximally entangled ones, and we construct RSP protocols exploiting the structure of the irreducible representations of Abelian groups.
\end{abstract}

\section{INTRODUCTION}

Quantum communication, i.e., the transfer of quantum information can involve three kinds of resources: entanglement, classical communication, and direct transfer of quantum information. Quantum communication protocols that make use of the entangled resource consist of a suitable measurement performed on the sender's half of the entangled pair, followed by classical communication. The measurement depends on the target state that is to be transmitted, and the classical message respects the measurement outcome. Essentially, this action can always be viewed as a remote state preparation (RSP) scheme 1, 2, 3, 4, 5, 6, 7, 8, 9, 10, 11, 12, 13. The goal of RSP is to prepare a given quantum state at a distant location using only shared entangled pairs and classical communication as resources.

An intriguing question about RSP is whether the relevant information describing the target state is transmitted over the classical communication channel 11, 12, 13. or the entangled resource [1, 2, 3, 4, 5, 6, 7, 8, 9]. In the latter case, no access to it can be gained from the classical message. Such protocols are called oblivious. This aspect is of crucial importance if, e.g., quantum communication is introduced for securing a private communication channel. Therefore, it is worth to investigate in what conditions an RSP protocol is oblivious. In this Paper, we give a necessary and sufficient condition for a generic exact RSP protocol to be oblivious. A protocol is called exact (or faithful) if it succeeds with unit fidelity. For the existence of those deterministic exact RSP protocols in which the measurement eigenstates are related to each other by linear operators similar to Bob's unitaries, we provide a simple condition in terms of commutation relations. We give a method for constructing such protocols and trace them back to the case of maximally entangled resources.

The paper is organized as follows. In Sec. [1] we introduce a mathematical model of RSP and argue for the importance of antilinear operators in RSP. In Sec. III we prove the necessary and sufficient condition of obliviousness. Then we investigate exact deterministic protocols using partially entangled states and give an example how to reduce them to simpler ones in Sec. IV Finally, Sec. $\mathrm{V}$ summaries our results.

\section{ANTILINEARITY INVOLVED IN RSP}

We start from an RSP protocol in which the sender (Alice) and the receiver (Bob) share a pure but not necessarily maximally entangled state $|\Psi\rangle_{A B}$. The use of mixed resources would prevent the scheme from being able to exactly prepare a pure state. Suppose that Alice performs a von Neumann measurement whose result is given by the rank one projection $|\phi\rangle_{A A}\langle\phi|$. Alternatively, it may be a rank one positive operator with $\|\phi\|^{2}<1$ for a POVM measurement. Due to the very nature of the von Neumann projection principle, Bob's unnormalized conditional state is an antilinear (conjugate linear) function $R: \mathcal{H}_{A} \rightarrow \mathcal{H}_{B}$ of Alice's measurement eigenstate

$$
|\psi\rangle_{B} \propto{ }_{A}\langle\phi \mid \Psi\rangle_{A B} \equiv R|\phi\rangle_{A},
$$

where the partial scalar product is antilinear in its first argument, and the norm square of the right hand side gives the probability of this measurement event,

$$
p=\left\|{ }_{A}\langle\phi \mid \Psi\rangle_{A B}\right\|^{2}=\| R|\phi\rangle_{A} \|^{2} .
$$

The entangled state $|\Psi\rangle_{A B}$ is completely given by $R$ that is mapping a possible measurement eigenstate in system $A$ to the corresponding state of system $B$ after the measurement. Formally, $R$ is nothing more than the composition of a complex conjugation (like time inversion) with a linear operator, and it is diagonal in the Schmidt basis. The isomorphism between pure entangled states and antilinear operators, and its application to quantum teleportation has been thoroughly investigated in Refs. 14, 15, 16]. Without loss of generality, we can assume that the Hilbert spaces of Alice and Bob 
are both $D$ dimensional, and that the shared entangled state has nonzero Schmidt coefficients only, that is, the antilinear operator defined in (1) is invertible.

A sufficient and necessary condition for the existence of exact deterministic RSP protocols was given in Ref. [5] . It was shown that a target state $\left|\psi_{0}\right\rangle_{B}$ can be prepared unconditionally if and only if there is such a set of unitary transformations $U_{m}$ under Bob's control and the probability distribution $p_{m}\left(\psi_{0}\right)$ for Alice's measurement is such that

$$
\sum_{m=0}^{N-1} p_{m}\left(\psi_{0}\right) U_{m}^{\dagger}\left|\psi_{0}\right\rangle_{B B}\left\langle\psi_{0}\right| U_{m}=\rho_{B}
$$

where $\rho_{B}$ is the partial density operator of the entangled state $|\Psi\rangle_{A B}$ and $N$ is the number of possible outcomes. (For a projective measurement $N=D$, while $N>D$ for a POVM.) Eq. (3) expresses the fact that the density operator of system $B$ is not changed by Alice's action as long as the outcome of the measurement is unspecified. Then the eigenstates of Alice's measurement can be constructed as

$$
\left|\phi_{m}\right\rangle_{A}=\sqrt{p_{m}\left(\psi_{0}\right)} R^{-1} U_{m}^{\dagger}\left|\psi_{0}\right\rangle_{B},
$$

where $R$ is the antilinear operator defined in (II) that describes the entangled resources. The set $\mathcal{E}_{B} \subset \mathcal{H}_{B}$ of states is called a preparable ensemble if it consists of states that can be prepared using the same fixed set of unitaries $U_{m}$.

If the exact deterministic RSP protocol is obliviousthat is, the probability distribution $p_{m}\left(\psi_{0}\right)$ is independent of the target states $\left|\psi_{0}\right\rangle_{B} \in \mathcal{E}_{B}$-then Eq. (4) implies that the eigenstates of Alice's measurement are antilinear functions of the target state: for each successful outcome $m$, there exists an operator $A_{m}: \mathcal{H}_{B} \rightarrow \mathcal{H}_{A}$ such that

$$
\left|\phi_{m}\right\rangle_{A}=A_{m}\left|\psi_{0}\right\rangle_{B}, \quad A_{m}=\sqrt{p_{m}} R^{-1} U_{m}^{\dagger},
$$

and $A_{m}$ is antilinear and is the same for all $\left|\psi_{0}\right\rangle_{B} \in \mathcal{E}_{B}$.

Let us verify the existence of such antilinear operators in two well-known examples. Quantum teleportation of a qubit state can be regarded as an oblivious RSP protocol. Alice performs a POVM measurement consisting of four one-rank positive operators. One of them is

$$
\left|\phi_{0}\right\rangle_{A A}\left\langle\phi_{0}\left|=\frac{1}{2} R^{-1}\right| \psi_{0}\right\rangle_{B B}\left\langle\psi_{0}\right| R^{-1 \dagger},
$$

with $\left\|\phi_{0}\right\|^{2}=1 / 2$ and $U_{0}=\mathbb{1}_{B}$ being the identity operator. The other three positive operators are $\left|\phi_{i}\right\rangle_{A A}\left\langle\phi_{i}\right|$ where the (unnormed) vectors $\left|\phi_{i}\right\rangle_{A}$ are obtained from $\left|\phi_{0}\right\rangle_{A}$ by the Pauli matrices. All the four eigenstates are related to the target state in an antilinear way, because $A_{0}=\frac{1}{2} R^{-1}$ and $A_{i}=\frac{1}{2} \sigma_{i} R^{-1}$.

Another kind of oblivious RSP protocol was introduced in Ref. 1] that needs half the amount of classical communication than teleportation. In this scheme, Alice performs a von Neumann projective measurement, one of its eigenstates being $\left|\phi_{0}\right\rangle_{A}=\frac{1}{\sqrt{2}} R^{-1}\left|\psi_{0}\right\rangle_{B}$ with $\left\|\phi_{0}\right\|^{2}=1$. The other eigenstate $\left|\phi_{1}\right\rangle_{A}$ is orthogonal (antipodal) to $\left|\phi_{0}\right\rangle_{A}$. For states $\left|\phi_{0}\right\rangle_{A}$ chosen from a special ensemble, namely, for states on a great circle of the Bloch sphere, the $\pi$-rotation with axis perpendicular to the circle is the unitary transformation $U$ that maps $\left|\phi_{0}\right\rangle_{A}$ to $\left|\phi_{1}\right\rangle_{A}$. Therefore, we have two antilinear operators $A_{0}=\frac{1}{\sqrt{2}} R^{-1}$ and $A_{1}=\frac{1}{\sqrt{2}} U R^{-1}$, that are antiunitary as well in this case. Unlike in quantum teleportation, the ensemble of preparable states $\mathcal{E}_{B}$ is restricted to a great (or small [2]) circle in this protocol.

We note that the POVM operators, like in Eq. (6), are linear functions of the matrix elements of the target density operator $\left|\psi_{0}\right\rangle_{B B}\left\langle\psi_{0}\right|$, since complex conjugation is equivalent to transposition for Hermitian matrices. The corresponding linear superoperators are, however, not completely positive.

Though the eigenstates of the POVM operators are necessarily antilinear functions of the target state, this fact does not exclude the possibility that, in some special cases, we can find linear operators $L_{m}: \mathcal{H}_{B} \rightarrow \mathcal{H}_{A}$ as well that also generate the eigenstates from the target state, $\left|\phi_{m}\right\rangle_{A}=L_{m}\left|\psi_{0}\right\rangle_{B}$. This can happen, for example, in the trivial case when $\mathcal{E}_{B}$ consists of a basis $|i\rangle_{B}$ and nothing more. Then we can define the linear operators such that $L_{m}|i\rangle_{B} \equiv A_{m}|i\rangle_{B}$. However, this kind of "classical RSP" is nothing more than a classical hashing protocol with entanglement providing the shared secret key (c.f. onetime pad), and it is out of the scope of the present Paper.

A similar construction of linear operators is also possible when $\mathcal{E}_{B}$ consists of real superpositions of the basis states. On this real subspace, complex conjugation does not play role, the antilinear $A_{m}$ equals to the linear $L_{m}$, so antilinearity is not an issue. However, as it is shown in Ref. [3], RSP is realizable in real Hilbert space only if the dimension of the space is 2,4 , or 8 . This means that $\mathcal{E}_{B}$ is usually not a real subspace, so complex conjugation does matter and antilinear functions are the simplest choice to relate measurement eigenstates to the target state.

\section{CONDITION OF OBLIVIOUSNESS}

We have seen in Sec. II that a necessary condition for an exact RSP scheme to be oblivious is that for all the outcomes $m$, either there exists a fixed antilinear operator $A_{m}$ mapping the target state $\left|\psi_{0}\right\rangle_{B}$ to the measurement eigenstate $\left|\phi_{m}\right\rangle_{A}$, or Bob registers the outcome $m$ as a failure. The question naturally arises whether the existence of such antilinear operators is a sufficient condition of obliviousness. In this section, we show that the answer is positive.

Suppose that we are given an exact RSP protocol in which there exist such antilinear operators $A_{m}$, but we do not require that the protocol is oblivious. We utilize the result of [5] that it suffices for Bob to perform a unitary operation in order to restore the target state. (Actually, 
Ref. [5] proves it in the case when $\mathcal{E}_{B}=\mathcal{H}_{B}$, but with a slight modification of the proof it is easy to see that it holds for arbitrary preparable ensemble.) If $U_{m}$ denote the unitary operation corresponding to a successful outcome $m$, then the corresponding measurement eigenstate is necessarily

$$
\left|\phi_{m}\right\rangle_{A}=\sqrt{p_{m}\left(\psi_{0}\right)} R^{-1} U_{m}^{\dagger}\left|\psi_{0}\right\rangle_{B}
$$

If the measurement yields the result $m$, Bob's state becomes

$$
\left|\psi_{m}\right\rangle_{B}=\frac{1}{\sqrt{p_{m}\left(\psi_{0}\right)}} R A_{m}\left|\psi_{0}\right\rangle_{B}
$$

The probability $p_{m}\left(\psi_{0}\right)=\| R A_{m}\left|\psi_{0}\right\rangle_{B} \|^{2}$ of this outcome may depend on the target state $\left|\psi_{0}\right\rangle_{B}$ if the protocol is not oblivious. After Bob applied the unitary operation $U_{m}$, his state must agree with the target state, so after rearranging (8), we have

$$
U_{m} R A_{m}\left|\psi_{0}\right\rangle_{B}=\sqrt{p_{m}\left(\psi_{0}\right)}\left|\psi_{0}\right\rangle_{B}, \quad \text { for all }\left|\psi_{0}\right\rangle_{B} \in \mathcal{E}_{B}
$$

and for all outcome $m$ that does not correspond to a failure. The left hand side of the equation is linear in $\left|\psi_{0}\right\rangle_{B}$ since $A_{m}$ and $R$ are both antilinear and their product is linear. Therefore, the right hand side must be linear as well - as long as the superposition states used to test its linearity are also elements of $\mathcal{E}_{B}$. We show now that this implies obliviousness.

To this end, we pose an important assumption regarding the ensemble $\mathcal{E}_{B}$. We will refer to it as the assumption of "sufficiently large ensemble." We assume that $\mathcal{E}_{B}$ consists states that are not linearly independent, e.g., there is a particular nontrivial superposition state of some other vectors that form a basis in $\mathcal{E}_{B}$. From the practical point of view, the ensemble $\mathcal{E}_{B}$ is not much restricted by this assumption. It expresses that we are not limited to a classical alphabet but $\mathcal{E}_{B}$ contains many states that are evidently not all orthogonal to each other. Essentially, nonorthogonal states are the starting point of many quantum information processing protocols. For the qubit case, our assumption is satisfied if $\mathcal{E}_{B}$ contains at least three states.

Let $|k\rangle_{B}$ denote a (not necessarily orthogonal) basis in $\mathcal{E}_{B}$. Then $\left|\psi_{0}\right\rangle_{B}$ can be expanded as $\left|\psi_{0}\right\rangle_{B}=\sum_{k} c_{k}|k\rangle_{B}$. The linearity of the right hand side of Eq. (9) means:

$$
\begin{gathered}
\sqrt{p_{m}\left(\psi_{0}\right)}\left|\psi_{0}\right\rangle_{B}=\sum_{k} c_{k}\left(\sqrt{p_{m}(k)}|k\rangle_{B}\right), \\
0=\sum_{k} c_{k}\left(\sqrt{p_{m}(k)}-\sqrt{p_{m}\left(\psi_{0}\right)}\right)|k\rangle_{B} .
\end{gathered}
$$

Since the vectors $|k\rangle_{B}$ are linearly independent, only the trivial linear combination of theirs can be zero, so

$$
p_{m}\left(\psi_{0}\right)=p_{m}(k) \text { if } c_{k} \neq 0 .
$$

Following our assumption, if there is a particular superposition state $\left|\psi_{0}^{*}\right\rangle_{B}$ in $\mathcal{E}_{B}$ such that all the $c_{k}$ coefficients are nonzero, then (12) implies that $p_{m}(k)$ is the same number for every $k$. Furthermore, $p_{m}\left(\psi_{0}\right)$ for a general $\left|\psi_{0}\right\rangle_{B} \in \mathcal{E}_{B}$ has no state dependence either, that is, the protocol is oblivious. Thus we have concluded that for an exact RSP protocol that is capable of preparing a sufficiently large ensemble of target states, obliviousness is equivalent to that the measurement eigenstates $\left|\phi_{m}\right\rangle_{A}$ can be obtained from the target state using a fixed set of antilinear operators $A_{m}$.

\section{EXACT DETERMINISTIC RSP SCHEMES}

In the following, we investigate a special kind of oblivious exact deterministic RSP protocols, and we derive a simple condition for their existence in terms of commutation relations.

If an exact RSP protocol is deterministic (i.e., the "failure event" is excluded), then the condition of obliviousness given in Sec. III can be reduced to a similar condition in terms of linear operators: the scheme is oblivious if and only if the measurement eigenstates $\left|\phi_{m}\right\rangle_{A}$ are linear functions of the particular eigenstate $\left|\phi_{0}\right\rangle_{A}$, that is, $\left|\phi_{m}\right\rangle_{A}=L_{m}\left|\phi_{0}\right\rangle_{A}$ with fixed linear operators

$$
L_{m}=\sqrt{p_{m}\left(\psi_{0}\right) / p_{0}\left(\psi_{0}\right)} R^{-1} U_{m}^{\dagger} U_{0} R .
$$

The proof is similar to that in Sec. III the ratio $p_{m}\left(\psi_{0}\right) / p_{0}\left(\psi_{0}\right)$ is independent of $\left|\psi_{0}\right\rangle_{B}$, then the probabilities sum up to the probability of success that is 1 .

Let us suppose first that the operators $L_{m}$ in (13) are proportional to unitaries, i.e., $L_{m}^{\dagger} L_{m}=\ell_{m} \mathbb{1}_{A}$. Then the protocol is oblivious and, exploiting that the ratio $p_{m} / p_{0}$ is constant, we can rewrite (13) as

$$
R L_{m}=\sqrt{p_{m} / p_{0}} U_{m}^{\dagger} U_{0} R
$$

Multiplying (14) with its adjoint, taking its trace, and using that $R^{\dagger} R=\rho_{A}$ and $R R^{\dagger}=\rho_{B}$ are the reduced density operators of the entangled resource, we find that $\ell_{m}=p_{m} / p_{0}$ and (14) then implies the commutation relations

$$
\left[\rho_{B}, U_{0}^{\dagger} U_{m}\right]=0, \quad \text { for all } m,
$$

and similarly $\left[\rho_{A}, L_{m}\right]=0$.

We mention that the reverse is also true: The operators $L_{m}$ that map a particular measurement eigenstate $\left|\phi_{0}\right\rangle_{A}$ to the other eigenstates $\left|\phi_{m}\right\rangle_{A}$ are proportional to unitaries if and only if the commutation relation (15) holds. Then Alice's $L_{m}$ and Bob's $U_{m}^{\dagger} U_{0}$ are given in the Schmidt bases by matrices proportional to each other.

An important implication of this theorem is that if (15) holds for a general exact deterministic RSP scheme using partially entangled resources, then the same set of unitaries (at Bob's side) and the same measurement device (at Alice's side) with the same probability distribution can be used to remotely prepare a different ensemble using a maximally entangled resource. To show this, we 
start from the necessary and sufficient condition of RSP given in Eq. (3). Multiplying it with $\left(D \rho_{B}\right)^{-1 / 2}$ from both left and right we obtain

$$
\sum_{m=0}^{N-1} p_{m} U_{m}^{\dagger}\left|\psi^{\prime}\right\rangle_{B B}\left\langle\psi^{\prime}\right| U_{m}=\frac{1}{D} \mathbb{1}_{B}, \quad \text { for all }\left|\psi^{\prime}\right\rangle_{B} \in \mathcal{E}_{B}^{\prime},
$$

where now the preparable ensemble $\mathcal{E}_{B}^{\prime}$ contains all the dependence on the original partially entangled resource,

$$
\mathcal{E}_{B}^{\prime}=\frac{1}{\sqrt{D}} U_{0} \rho_{B}^{-1 / 2} U_{0}^{\dagger} \mathcal{E}_{B} \quad \text { and } \quad \mathcal{E}_{B}=U_{0} \sqrt{D \rho_{B}} U_{0}^{\dagger} \mathcal{E}_{B}^{\prime}
$$

We see that Eq. (16) is the necessary and sufficient condition of RSP using a maximally entangled resource. Note that for a maximally entangled resource the commutation relation (15) is trivially satisfied.

Conversely, protocols designed for maximally entangled resources can be used with partially entangled resources if and only if (15) holds with the partial density operator of that partially entangled resource. This observation can help us construct RSP protocols, since it is much easier to first seek for maximally entangled schemes that satisfy (16), and then test condition (15), while solving (3) directly can be difficult.

An interesting implication of the commutation relation (15) is that the unitary operators $U_{0}^{\dagger} U_{m}$ are simultaneously block diagonal in the eigenbasis of the partial density operator. $\rho_{B}$ can always be written as a linear combination of its eigenprojections,

$$
\rho_{B}=\sum_{j=1}^{r} \lambda_{j} P_{j}=\bigoplus_{j=1}^{r} \lambda_{j} \mathbb{1}_{j},
$$

where $r$ is the number of different eigenvalues of $\rho_{B}, P_{j}$ is the projection onto the eigensubspace $\mathcal{H}_{j}, \mathbb{1}_{j}$ is the identity operator on $\mathcal{H}_{j}$, and $\bigoplus$ denotes the direct sum of the operators. Suppose that in the lower dimensional subspaces the RSP problem (16) is solved for maximally entangled resources. Let us denote by $U_{m}^{(j)}$ the unitaries, by $p_{m}^{(j)}$ the probabilities, and by $\mathcal{E}_{j}$ the preparable ensemble that correspond to the RSP protocol in $\mathcal{H}_{j}$,

$$
\sum_{m=0}^{N_{j}-1} p_{m}^{(j)} U_{m}^{(j)}{ }^{\dagger}\left|\psi^{(j)}\right\rangle_{j j}\left\langle\psi^{(j)}\left|U_{m}^{(j)}=\frac{1}{D_{j}} \mathbb{1}_{j}, \quad\right| \psi^{(j)}\right\rangle_{j} \in \mathcal{E}_{j}
$$

Now we give a method for constructing an RSP protocol in $\mathcal{H}_{B}$. We define the block diagonal unitaries as

$$
U_{k, \mathbf{m}} \equiv \bigoplus_{j=1}^{r} e^{2 \pi i j k / r} U_{m_{j}}^{(j)}
$$

where $(k, \mathbf{m})$ indexes an outcome of the protocol to be constructed, with $k=1, \ldots, r$ and $\mathbf{m}=\left(m_{1}, \ldots, m_{r}\right)$ where $m_{j}$ indexes a possible measurement outcome of the RSP protocol in the subspace $\mathcal{H}_{j}$. We will not necessarily consider every combination of the $m_{j}$-s, but we require in our construction that the probabilities $p_{k, \mathbf{m}}=p_{\mathbf{m}}$ do not depend on $k$ and that

$$
r \sum_{\mathbf{m}} p_{\mathbf{m}} \delta_{n m_{j}}=p_{n}^{(j)}
$$

Then the following target states can be prepared remotely,

$$
\left|\psi_{0}\right\rangle_{B} \equiv \bigoplus_{j=1}^{r} \sqrt{\lambda_{j} D_{j}} e^{i \varphi_{j}}\left|\psi^{(j)}\right\rangle_{j}, \quad\left|\psi^{(j)}\right\rangle_{j} \in \mathcal{E}_{j},
$$

where $\varphi_{j}$-s are the RSP parameters that are specified by Alice and unknown to Bob. Neglecting the global phase factor of $\left|\psi_{0}\right\rangle_{B}$, there are $r-1$ free of them. If $\mathcal{E}_{j}$ is a $d_{j}$ dimensional real manifold, the dimensionality of $\mathcal{E}_{B}$ is $d=\sum_{j} d_{j}+r-1$.

To prove that our construction indeed realizes RSP, let us substitute $\left|\psi^{\prime}\right\rangle_{B}=\bigoplus_{j=1}^{r} \sqrt{D_{j} / D} e^{i \varphi_{j}}\left|\psi^{(j)}\right\rangle_{j}$ and (20) in the reduced RSP condition (16). Verifying it block by block, we immediately find that the off-diagonal blocks vanish because

$$
\frac{1}{r} \sum_{k=1}^{r} e^{2 \pi i(l-j) k / r}=\delta_{j l}
$$

The RSP condition for the remaining diagonal blocks then reduces to Eq. (19) which was our starting point.

Before we present a concrete example, we note that the index $k$ is an element of the cyclic group $\mathbb{Z}_{r}$ that is the group of integer addition modulo $r$. This can be straightforwardly generalized to an arbitrary Abelian group $G$ of order $r$. It is known from group theory that an Abelian group $G$ is isomorphic to a direct product of cyclic groups,

$$
G \simeq \mathbb{Z}_{r_{1}} \times \mathbb{Z}_{r_{2}} \times \ldots \times \mathbb{Z}_{r_{p}} .
$$

Evidently, $|G|=\prod_{i=1}^{p} r_{i}=r$. Elements of $G$ can be treated as $p$-tuples $\mathbf{k}=\left(k_{1}, k_{2}, \ldots, k_{m}\right)$ with $k_{i}=0,1$, $\ldots, r_{i}-1$. Every element forms a conjugacy class onto itself, therefore, all the irreducible representations of $G$ are one dimensional, they are indexed by the group elements $(\mathbf{j} \in G)$, and they are products of irreducible representations of the respective cyclic groups,

$$
u_{\mathbf{j}}: G \rightarrow \mathbb{C}, \quad u_{\mathbf{j}}(\mathbf{k})=\prod_{i=1}^{p} \exp \left(2 \pi i j_{i} k_{i} / r_{i}\right)
$$

The one dimensional representations are the characters of themselves and Eq. (23) can be replaced by the following orthogonality relation of the characters of the irreducibles

$$
\frac{1}{|G|} \sum_{\mathbf{k} \in G} u_{\mathbf{j}}(\mathbf{k}) u_{\mathbf{l}}^{*}(\mathbf{k})=\delta_{\mathbf{j} \mathbf{l}}, \quad \text { for } \mathbf{j}, \mathbf{l} \in G
$$


We can redefine the block diagonal unitaries (20) as

$$
U_{\mathbf{k}, \mathbf{m}} \equiv \bigoplus_{\mathbf{j} \in G} u_{\mathbf{j}}(\mathbf{k}) U_{m_{\mathbf{j}}}^{(\mathbf{j})}
$$

and we find that the preparable ensemble $\mathcal{E}_{B}$ consists of states of the form

$$
\left|\psi_{0}\right\rangle_{B} \equiv \bigoplus_{\mathbf{j} \in G} \sqrt{\lambda_{\mathbf{j}} D_{\mathbf{j}}} e^{i \varphi_{\mathbf{j}}}\left|\psi^{(\mathbf{j})}\right\rangle_{\mathbf{j}}, \quad\left|\psi^{(\mathbf{j})}\right\rangle_{\mathbf{j}} \in \mathcal{E}_{\mathbf{j}}
$$

where the variables $\varphi_{\mathbf{j}}$ are the RSP parameters.

Now we underpin our construction by a simple example. Consider the RSP of a qutrit state where the entangled resource has two different eigenvalues so that $\rho_{B}=\operatorname{diag}\left(\lambda_{1}, \lambda_{1}, \lambda_{2}\right)$ in the Schmidt basis. We take the equatorial RSP protocol of Ref. [1] in $\mathcal{H}_{1}$, so we have $p_{0}^{(1)}=p_{1}^{(1)}=\frac{1}{2}, U_{0}^{(1)}=\mathbb{1}, U_{1}^{(1)}=\sigma_{z}$, and $\mathcal{E}_{1}=\left\{\left(|0\rangle+e^{i \varphi}|1\rangle\right) \sqrt{2} \mid \varphi \in[0,2 \pi)\right\}$. For the one dimensional subspace $\mathcal{H}_{2}$ we take the trivial protocol $p_{0}^{(2)}=1$, $U_{0}^{(2)}=\mathbb{1}$, and $\mathcal{E}_{2}=\{|2\rangle\}$. We construct the qutrit RSP protocol to have 4 possible outcomes indexed by $(k, \mathbf{m})$ with $k=1,2$ and $\mathbf{m}=(0,0),(1,0)$. It can be easy checked that the choice $p_{k, \mathbf{m}} \equiv \frac{1}{4}$ for the new probabilities satisfies Eq. (21). Then Eq. (20) provides us with the unitary matrices

$$
\begin{array}{ll}
U_{1,(0,0)}=\operatorname{diag}(-1,-1,1), & U_{2,(0,0)}=\operatorname{diag}(1,1,1), \\
U_{1,(1,0)}=\operatorname{diag}(-1,1,1), & U_{2,(1,0)}=\operatorname{diag}(1,-1,1) .
\end{array}
$$

For the target states, Eq. (22) gives

$$
\left|\psi_{0}\right\rangle_{B}=\sqrt{\lambda_{1}}|0\rangle+\sqrt{\lambda_{1}} e^{i \varphi}|1\rangle+\sqrt{\lambda_{2}} e^{i \varphi^{\prime}}|2\rangle,
$$

where the RSP parameter $\varphi$ originates in the RSP protocol in $\mathcal{H}_{1}$, while $\varphi^{\prime}$ comes from our construction. Note that it generally holds that if we build up our protocol from the generalized equatorial protocols of [2], then we obtain the same preparable ensemble as one would obtain using the protocol of [2] directly - though with different measurement setup and unitaries.

\section{SUMMARY}

We have considered general oblivious RSP protocols. We have presented a necessary condition for a protocol to be oblivious: Alice's measurement eigenstates must be antilinear functions of the target state. We have pointed out the importance of antilinearity by some examples. We have shown that our antilinear condition of obliviousness is also sufficient if the ensemble of preparable target states is sufficiently large. For qubits, it means that the ensemble contains more than two states.

We have also considered exact deterministic RSP protocols in which the operators that map the measurement eigenstates to each other are similar to Bob's unitaries. We have derived conditions for the existence of such protocols in terms of commutation relations. We have shown that they can be traced back to protocols using maximally entangled resources and, therefore, our conditions are easy to use even if we have nonmaximally entangled resources. To underpin it, we have constructed a protocol from subprotocols given in the eigensubspaces of the reduced density matrix of the partially entangled resource.

\section{Acknowledgments}

This work was supported by the National Research Fund of Hungary under contracts T043287 and T049234, and by the Marie Curie Programme of the European Commission (MERG-CT-2004-500783). One of the authors thanks A. K. Pati for useful discussions on RSP.
[1] A. K. Pati, Phys. Rev. A 63, 014302 (2000).

[2] M.-Y. Ye, Y.-S. Zhang, and G.-C. Guo, Phys. Rev. A 69, 022310 (2004).

[3] B. Zeng and P. Zhang, Phys. Rev. A 65, 022316 (2002).

[4] D. W. Leung and P. W. Shor, Phys. Rev. Lett. 90, 127905 (2003).

[5] A. Hayashi, T. Hashimoto, and M. Horibe, Phys. Rev. A 67, 052302 (2003).

[6] C. H. Bennett, D. P. DiVincenzo, P. W. Shor, J. A. Smolin, B. M. Terhal, and W. K. Wootters, Phys. Rev. Lett. 87, 077902 (2001).

[7] C. H. Bennett, P. Hayden, D. W. Leung, P. W. Shor, and A. Winter, IEEE Trans. Inform. Theory 51, 56 (2005), quant-ph/0307100.

[8] Z. Kurucz, P. Adam, Z. Kis, and J. Janszky, Phys. Rev. A 72, 052315 (2005).

[9] M. Koniorczyk, Z. Kurucz, A. Gábris, and J. Janszky,
Phys. Rev. A 62, 013802 (2000).

[10] P. Agrawal, P. Parashar, and A. K. Pati, Int. J. Quant. Information 1, 301 (2003), quant-ph/0304006.

[11] I. Devetak and T. Berger, Phys. Rev. Lett. 87, 197901 (2001).

[12] A. Abeyesinghe and P. Hayden, Phys. Rev. A 68, 062319 (2003).

[13] D. W. Berry, Phys. Rev. A 70, 062306 (2004).

[14] A. Uhlmann, in Lecture Notes on Physics, edited by A. Borowiec (Springer-Verlag, Berlin, 2000), vol. 539, pp. 93-105, quant-ph/9901027, see also quant-ph/0301116 and quant-ph/0407244

[15] Z. Kurucz, M. Koniorczyk, and J. Janszky, Fortschr. Phys. 49, 1019 (2001), quant-ph/0308020.

[16] Z. Kurucz, M. Koniorczyk, P. Adam, and J. Janszky, J. Opt. B: Quantum Semiclass. Opt. 5, S627 (2003). 